\section{Arqueología de la Arquitectura en España}

JuAn Antonio Quirós CASTILLO ${ }^{1}$

Universidad del País Vasco / Euskal Herriko Unibertsitatea

\section{INTRODUCCIÓN}

El objetivo de esta ponencia será el de trazar un cuadro general sobre el concepto actual de la arqueología de la arquitectura en la Península Ibérica, y plantear una serie de propuestas y de elementos de reflexión que la disciplina tendrá que abordar en un próximo futuro.

El primer aspecto que es necesario discutir es precisamente el del concepto de arqueología de la arquitectura. A nalizando los contenidos de los últimos trabajos editados en España sobre está temática², es evidente que existe una concepción muy heterogénea del significado y el campo de actuación de la denominada arqueología de la arquitectura.

$\mathrm{H}$ an sido acogidos dentro de este concepto lecturas estratigráficas de alzados, el análisis de materiales constructivos antiguos, el estudio del área de captación de estos materiales, propuestas de intervención en ocasión de restauraciones y rehabilitaciones de edificios, análisis arqueométricos, lecturas basadas en criterios analógicos y estilísticos, la excavación de las bóvedas de iglesias, propuestas de instrumentos de datación, el estudio de procesos sociales a partir del documento arquitectónico, etc. Frente a esta notable diversidad, es oportuno reflexionar sobre el significado del concepto y el uso que se hace de él.

El término de arqueología de la arquitectura fue acuñado hace ya más de diez años en Italia (MANNONI, 1990: 28), y surgió con el fin de agrupar toda una serie de experiencias e investigaciones realizadas en los dos decenios anteriores como resultado de la aplicación de los instrumentos, conceptos y problemáticas de la disciplina arqueológica al estudio de la arquitectura.

N aturalmente, los arqueólogos se habían ocupado con anterioridad de la arquitectura, y existe una larga tradición de estudios que se remonta a los al bores mismos de la disciplina arqueológica. Basta recordar, por ejemplo, los importantes estudios real izados por la arqueol ogía clásica en la postguerra en los campos del estudio de las técnicas constructivas 0 , a partir de autores como N. Lamboglia (1958), en el empleo de la estratigrafía del subsuelo para fechar los monumentos. Por todo ello puede resultar

\footnotetext{
${ }^{1}$ Se agradecen a $P$. Latorre sus comentarios críticos al presente texto, y a J. Grenville, A. López Mullor, M. A. Tabales y L. Caballero sus sugerencias e indicaciones bibliográficas. Deseo expresar mi agradecimiento especial a Agustín Azkarate y a todo el Grupo de Investigación en A rqueología de la Arquitectura de la U niversidad del País Vasco, que me han dado numerosas informaciones e indicaciones para poder redactar la presente ponencia.

${ }^{2}$ Concretamente nos referimos a la sección "M etodología. A rqueología de la Arquitectura" del V Congreso de Arqueología M edieval Española celebrado en Valladolid, cuyas actas han visto la luz en el año 2001 (vol. 1: 177-414), así como los setenta posters presentados al Seminario Internacional de A rqueología de la Arquitectura celebrado en Vitoria-Gasteiz el mes de febrero del 2002, que se publicarán en el segundo número de esta misma revista.
} 
paradójico, e incluso pretencioso, adoptar una nueva terminol ogía para referirnos a los estudi os arqueológicos de la arquitectura. Como se ha señal ado recientemente, solo el nombre es nuevo; el estudio de la arquitectura se remonta a los orígenes mismos de la arqueología, en el Renacimiento italiano, por lo que, de hecho, es la más vieja de las arqueologías (MORRISS, 2000: 5).

Sin embargo, solamente en los últimos decenios se han empezado a emplear ciertos instrumentos (en primer lugar la lectura estratigráfica de al zados), a plantearse nuevos problemas y ámbitos de investigación hasta entonces inexplorados y, sobre todo, se ha desarrollado una práctica arqueol ógica orientada a la investigación aplicada.

A unque, como veremos, en el caso de España ha sido fundamental la influencia de las experiencias italianas, es preciso contextualizar el surgimiento de esta disciplina en un marco de referencia más amplio, en el que tengan cabida otro tipo de tradiciones europeas. D e hecho, en los últimos decenios se ha asistido en toda Europa a una profunda renovación de los estudios arquitectónicos realizados por arqueólogos, cuyo resultado ha sido el surgimiento de líneas de trabajo como la A rchéol ogie du bâti o A rchéol ogie des dévations en Francia (Pringent, Hun OT, 2000; EsQuieu, 1997; JOURNOT, 1999), la Bauforschung y la Ilamada "arqueología de la construcción" en Alemania (AA. VV., 1996; BeDAL, 1993; KIMPEL, 1995), o la A rchaeology of $B$ uil dings o B uilding A rchaeol ogy en G ran Bretaña (W O0D, 1994; M ORRISS, 2000; ROSK AMS, 2000: 107-134).

$D$ os son las razones fundamental es que se encuentran detrás de este renovado interés por la arquitectura en los últimos decenios; por un lado el notable desarrollo adquirido por una arqueol ogía implicada en el estudio y gestión del patrimonio edificado como recurso finito en el marco de intervenciones de restauración y rehabilitación de la arquitectura histórica; pero además ha sido también fundamental la renovación de la disciplina arqueológica, a través de su reconstrucción estratigráfica y la ampliación de sus clásicos marcos cronológicos y temáticos.

N o obstante, aunque todas estas líneas de trabajo son hijas de un mismo contexto social y científico, su base genética es muy distinta, así como su desarrollo y ámbito de aplicación, por lo que estamos muy lejos de poder hablar de una "disciplina" única. $\mathrm{Ni}$ siquiera a nivel instrumental existe una homogeneidad. Curiosamente al gunas de estas líneas de trabajo son aún pre estratigráficas y abordan el estudio de la arquitectura histórica exclusivamente con instrumentos analógicos, tipológicos y estilísticos heredados de la historia del arte y de la arquitectura.
D esde este punto de vista, la experiencia ital iana es la que cuenta con una masa crítica de estudios más afirmada, y una codificación y normalización de instrumentos y conceptos más sólida, basada en la adopción de la filología estratigráfica como criterio fundamental de análisis de la arquitectura (BROGIOLO, 2002).

Es precisamente desde I tal ia desde donde la arqueología de la arquitectura se ha "importado" a la Península Ibérica. No debe extrañarnos esta estrecha relación con la arqueología italiana, ya que el peso que ha tenido en la historia reciente de la arqueología de época histórica española es muy notable. Como ha señal ado recientemente $X$. Dupré, ha sido precisamente a partir de las experiencias italianas y británicas como se ha producido la alfabetización estratigráfica española. Primero a través de la participación del ya mencionado Lamboglia en los cursos de A mpurias, y posteriormente -y de forma paralela a los contactos y a la presencia en España de arqueól ogos ingleses- como filtro que ha consentido la adquisición de los logros estratigráficos británicos en España y la introducción del llamado "sistema Harris" (DupRÉ, 1997: XIV).

Teniendo en cuenta estas circunstancias, puede ser útil plantear al gunos criterios básicos que contribuyan a definir la arqueología de la arquitectura:

1. La arqueología de la arquitectura es una disciplina arqueológica, y por lo tanto, como disciplina histórica que es, persigue el conocimiento de la sociedad a través de los documentos materiales, en este caso arquitectónicos. $\mathrm{N}$ o la concebimos, pues, sólo como un instrumento para conocer la historia del edificio o para replantarse la historia de la arquitectura.

2. Otro segundo aspecto que define la disciplina es el compromiso con el estudio y la gestión del patrimonio edificado. Desde este punto de vista la concebimos, no sólo como investigación básica, sino también aplicada. Es preciso tener en cuenta el carácter bidimensional del Patrimonio edificado, en cuanto siendo documento de las sociedades pasadas, es también recurso para las sociedades actuales. Este es un criterio básico que diferencia la arqueología de la arquitectura de otros estudios arquitectónicos real izados por arqueól ogos.

3. El tercer criterio de referencia es el empleo de un bagaje instrumental de naturaleza estrictamente arqueológica. En España, como en el resto de los estados de nuestro entorno, ha sido y sigue siendo el lenguaje estratigráfico el medio de expresión de la arqueología del final del siglo XX y de inicios del XXI. Pero además de la estratigrafía se han desarrollado una serie de instrumentos 
arqueológicos que permiten fechar y analizar con rigor el documento arquitectónico.

4. La arqueología de la arquitectura pretende situarse en una incómoda posición disciplinar intermedia que supere los límites tradicionales entre la arqueología, la arqueometría, la restauración y la arquitectura. En tradiciones consolidadas, como la italiana, es posible observar cómo se ha llegado a la socialización del instrumental e incluso de las perspectivas de trabajo e investigación, aunque este diálogo disciplinar no está exento de problemas.

5. Por último, se propugna el desarrollo de modelos interpretativos que, desde posiciones antropológicas, funcionalistas o materialistas, se contrapongan a los presupuestos ideal istas y positivistas profundamente arraigados en la historiografía de la arquitectura. A este propósito, no deja de ser significativo el impulso que ha ofrecido la arqueología de la arquitectura a la construcción de una historia social dela producción arquitectónica en el último decenio, y que haya cuestionado abi ertamente aspectos claves de la teoría de estil os vigente en el estudio de la arquitectura postclásica.

\section{LA ARQUEOLOGÍA DE LA ARQUITECTURA EN EL ÚLTIMO DECENIO}

Pero para comprender mejor cual es la situación de la "disciplina" en la actualidad, es preciso analizar brevemente las etapas formativas de lo que llamamos arqueología de la arquitectura. No es nuestro objetivo realizar una historiografía completa del decenio largo de experiencias acumuladas, sino que se pretende sencillamente resaltar algunas tendencias principales. A fines expositivos, es posible diferenciar dos fases principal es:

\section{Primera fase: hasta el año 1995}

Una primera fase que podemos situar desde los años 80 hasta mediados de los 90 se caracteriza por la experimentación y la búsqueda de nuevos instrumentos y criterios arqueológicos orientados al estudio de la arquitectura.

Como resultado de la puesta en marcha del sistema autonómico y de la descentralización de la gestión del patrimonio histórico, se ha producido durante estas décadas una explosión descontrolada de intervenciones arqueológicas como apoyo a la rehabilitación y restauración de edificios históricos. Es en este seno en el que se experimentarán nuevas vías de estudio del patrimonio arquitectónico.
$\mathrm{N}$ ormalmente la gran parte de los trabaj os experimentales realizados en este período se desarrollaron desde una perspectiva estrictamente de investigación, aunque pronto se intuyó las posibilidades de su desarrollo en el ámbito de la gestión.

Un ejemplo de esta fase experimental está representado por los trabajos realizados en Burgos por $\mathrm{J}$. A. A paricio (1990), que, sin llegar a formalizar un sistema conceptual y de registro estrictamente estratigráfico, intuyó la necesidad de establecer las relaciones indirectas de los distintos el ementos anal izados, sistematizó las relaciones estratigráficas, y cuestionó las dataciones estilísticas.

Otras experiencias relevantes fueron realizadas en esos mismos años por el equipo arqueológico de la Diputación de Barcelona, que fue asimilando progresivamente la dimensión arqueológica del edificio y decantando sus instrumentos de análisis. De esta manera, ya a partir de los años 80 se logró codificar un protocolo de intervención estratigráfica integral de los edificios analizados (LóPEZ MULLOR, 2002).

Se podrían señalar varios casos más de autores implicados en la búsqueda de nuevas estrategias de estudio de la arquitectura o preocupados en la función del arqueólogo en el marco de la rehabilitación y restauración monumental que han configurado el caldo de cultivo sobre el que se ha sedimentado, ya a partir de los primeros años 90, las primeras aplicaciones que podemos calificar de arqueología de la arquitectura (SOUTO LASALA, 1986; lópez Mullor, 1994; Castillo Armenteros et alii, 1999).

De forma paralel a a esta fase experimental propiciada por el nuevo marco de actuación de la "arqueología involuntaria" o "de gestión", se han producido otros dos hechos que merecen ser señal ados.

Por un lado es rel evante señal ar cómo una parte de los arquitectos restauradores han visto en este nuevo marco administrativo una oportunidad para integrar de forma rigurosa las informaciones estratigráficas, entendiendo la acción del arqueólogo como una ventaja y no como un inconveniente. A este propósito se pueden señalar trabajos como los de A. Jiménez realizados en Sevilla, la praxis impuesta por A. González desde la Diputación de Barcelona, 0 las intervenciones de P. Latorre y L. Cámara en M elque, Faro de la Torre de H ércules de Coruña, iglesia de San Pedro el Viejo de Arlanza, por señalar algunos ejemplos concretos. A simismo es relevante subrayar la labor de A. Almagro en la aplicación de la fotogrametría (p.e. Almagro et alii, 1992). 
Es asimismo rel evante señalar la renovación que ha tenido lugar en estos decenios en el estudio de la arquitectura del mundo clásico, tanto a través del desarrollo de sistemas de lectura y análisis que han superado los planteamientos clásicos (sólo por poner un ejemplo, el estudio del arco de Bará en Tarragona se ha realizado siguiendo una lógica estratigráfica, aunque no se ha formal izado la fil ol ogía harrisiana, DUPRÉ, 1994: 137-201), como en el estudio de los materiales y las técnicas constructivas (p.e. BENDALA, GALÁN, 1992; RODȦ, 1994), por señalar solamente al gunas de las problemáticas principales.

En este contexto ha representado un sal to notable la renovación instrumental representada por la "importación" desde Italia a partir de los años 90 de los conceptos e instrumentos que consideramos bási cos de la arqueología de la arquitectura.

El principal responsable de esta "importación" ha sido Luis Caballero, que ha ejercido como un verdadero canal de transmisión entre las dos tradiciones arqueológicas. Su acercamiento a la arqueología de la arquitectura se ha real izado con el fin de dotarse de un instrumento analítico de mayor capacidad y rigor para el estudio de la arquitectura al tomedieval peninsular. De hecho, sus numerosas intervenciones arqueológicas en iglesias de época al tomedieval han conseguido renovar el conocimiento sobre la arquitectura de este período y replantear su estudio sobre nuevas bases (CABALLERo ZOREDA, 1994-1995).

A diferencia de estas experiencias, se va a gestar un grupo en la Universidad del País Vasco dirigido por Agustín Azkarate que, concentrando su actividad en el ámbito del País Vasco, marcará como criterios prioritarios la integración entre investigación básica y aplicada a la gestión y puesta en valor del patrimonio edificado en el marco de convenios con las administraciones implicadas. En este contexto, se va a primar una estrategia de intervención territorial, actuando sobre conjuntos arquitectónicos de distinta entidad y características, que comprenden edificios religiosos rurales, puentes, arquitectura civil, o incluso grandes conjuntos monumentales (AzKARATE, 2002).

También en los primeros años 90 se llevaron a cabo otras experimentaciones en varios lugares de España, entre los que se pueden señalar a modo de ejemplo los realizados en Cuenca (Coll ConesA \& alii, 1992), en el castillo de Gelide (Galindo Torres et alii, 1994) o en Sevilla, a partir de los trabajos en el Palacio de Mañara y, sobre todo, en el Convento de Santa M aría de los Reyes, por señalar algunos de los trabajos más significativos (TABALES, 1999).

\section{Segunda fase: desde el año 1995}

En el año 1995 se publica en una revista de arquitectura un número monográfico dedicado a la arqueología de la arquitectura bajo el título "Leer el documento construido" (CABallero ZOREDA, LATORRE, 1995). Esta iniciativa precede en varios meses la cel ebración de un curso en Burgos (CAballero Zoreda, Escriban 0, 1996), en el que se amplían algunos de los presupuestos enunciados en la anterior publicación ${ }^{3}$.

Ambas publicaciones representan el esfuerzo más serio real izado hasta el momento de sistematizar y normativizar los criterios que habían guiado las intervenciones real izadas en la Península I bérica.

El impacto de estos dos trabaj os ha sido muy notable, puesto que se agotaron en pocos meses. Estas publicaciones sirvieron, además, para que se diesen a conocer los trabajos de autores italianos como R. Parenti (1995, 1996, 1997) o G. P. Brogiolo (1995), traducidos al castellano para la ocasión.

Paral elamente se realizaron otras reuniones (p.e. "EI monument, document", Barcelona, 1995), aumentó el número de publicaciones temáticas y se ha llevado a cabo un importante número de experiencias en toda España que ha dado lugar, en al gunos casos, a la consol idación de equipos y grupos de trabajo, 0 a la puesta en marcha de distintos programas de trabajo.

En el año 1996 se publica en Portugal el primer trabajo dedicado al argumento (MAGalHaES RAMALHo, 1996), y también en los años siguientes empiezan a realizarse lecturas estratigráficas en Cuba o M éxico inspiradas en experiencias europeas.

No hemos querido referirnos en esta síntesis a ninguna intervención específica - ni siquiera al complejo estudio sobre la Catedral de Santa M aría de Vitoria- ya que en otras ponencias publicadas en esta misma revista se recogen de forma puntual las principal es experiencias realizadas en los últimos años.

Es llamativo observar que las críticas que se han producido contra la arqueología de la arquitectura no proceden de arquitectos, sino de arqueól ogos que cuestionan la propia necesidad de la "disciplina". Entre estas críticas se ha señalado la incapacidad de discernir en las secuencias estratigráficas distintas fases cronológicas debido a "los

\footnotetext{
${ }^{3}$ Con anterioridad se había celebrado en Salobreña una reunión dedicada a la "Arqueología del Monumento" en el año 1992, pero su tardía publicación y su escasa difusión ha limitado notablemente su influencia (M ALPICA, 1999), a pesar de los importantes estudios que realizan sobre esta materia el equipo de $A$. $M$ al pica sobre aspectos como las técnicas constructivas y la arquitectura islámiCa (M ALPICA, 1999).
} 
replanteos de obra, la torpeza de los ejecutantes o la simple necesidad de resolver situaciones imprevistas", o se ha cuestionado la oportunidad de recurrir a diagramas estratigráficos (García de CASTRo VALDÉs, 1997).

Pero al margen de estas críticas puntual es y circunscritas de carácter reaccionario, lo que si es cierto es que la arqueología de la arquitectura en la Península I bérica es una disciplina en franca expansión y que no se circunscribe a especial istas de un período arqueológico concreto. Las intervenciones ya realizadas en edificios de época romana, - los trabajos de prehistoriadores que se identifican bajo el epígrafe de la arqueología de la arquitectura (SÁn CHEZ, 1998) son buena prueba de ello.

Es rel evante señal ar asimismo que la fil ología estratigráfica ha sido adoptada por equipos de investigación formados por profesional es como arqueól ogos, restauradores y arquitectos ${ }^{4}$, que permiten pensar que la arqueología de la arquitectura cuenta con las suficientes bases conceptuales y científicas como para adquirir un mayor protagonismo durante el decenio que acabamos de inaugurar.

\section{LA ARQUEOLOGÍA DE LA ARQUITECTURA COMO ARQUEOLOGÍA}

Una vez que se han planteado brevemente algunas cuestiones de carácter historiográfico, es preciso refl exionar sobre los aspectos hermenéuticos y conceptuales de la disciplina.

Como se ha señalado con anterioridad, uno de los pilares fundamentales de la arqueol ogía de la arquitectura es que ha conseguido desarrollar un bagaje instrumental y conceptual estrictamente arqueológico, bien diferenciado del empleado por otras disciplinas como la historia del arte 0 de la arquitectura.

Esto no quiere decir que sin estratigrafía de alzados no haya arqueología de la arquitectura, o que podamos identificar lectura estratigráfica de paramentos con toda la arqueol ogía de la arquitectura. Esta identificación, que en realidad es un reduccionismo de la disciplina a un instrumento concreto (BROGIOLO, 1997), es frecuente en las primeras formulaciones realizadas en España, cuando se hizo al usión a la arqueología de la arquitectura como un "método" (CAballero, Latorre, 1995), y se asoció estrechamente a la historia de las construcciones.

${ }^{4}$ Sin ánimo de exhaustividad, se pueden señal ar entre los últimos trabajos $A C I E ́ N$, 1999; Arce, 1999; Azuar et alii, 2000; Blanco, 2000; Caballero, Cámara, 1995; Cortazar, 2000; Escribano, 1999; Galindo et alii, 1994; Latorre, 1995; Lecanda, 2000; Menéndez Granda, 2000; Mileto, 2000; Sánchez, Dominguez, 2000; Tabales, 2000; Tudanca Casero, 2001; Cobos Guerra, 2000.
Es posible hacer buena arqueología realizando lecturas arqueológicas a partir del estudio de las modulaciones, como en el caso del Puente de M érida (Felj 00, 1997), estudiando la distribución de las marcas de cantero, como en el caso del monasterio cisterciense de Santa María de Armenteira en Pontevedra (Valle Pérez, 1988), o con análisis arqueométricos, como en el caso del conjunto de Torre de Palma en Portugal (M ALONEY, RINGBOM, 2000). Incluso se ha podido ver cómo el recurso a los instrumentos tipológicos continúe si endo válido, aunque sea necesario recurrir a tipologías "domesticadas" por la lógica estratigráfica y por el conocimiento de las estructuras de producción que sustentan la actividad arquitectónica (FerRando et alii, 1989).

Sin embargo, las experiencias arqueológicas realizadas en España en el último decenio, y en Italia desde los años 70 , han mostrado que la lectura estratigráfica es el instrumento más riguroso con el que contamos hasta el momento para realizar un análisis diacrónico de la arquitectura histórica. A este propósito es revelador que se realicen con una cierta frecuencia este tipo de lecturas en Gran Bretaña, Australia o en los Estados Unidos de $\mathrm{N}$ orteamérica. Sin embargo, la adaptación de la estratigafía a los alzados y el desarrollo de procedimientos y estrategias adecuadas es uno de los principal es campos de discusión en lugares como en Francia (JOURNOT, 1999; Esquieu, 1997: 133; Rouger, 1998), o en Gran Bretaña, de tal manera que aún se están experimentando y poniendo a punto las estrategias más adecuadas.

En este último país, de hecho, no todos los autores aceptan la posibilidad de utilizar los instrumentos de lectura estratigrafica a los al zados sin llevar a cabo una adaptación (GRENVILLE, 1997: 2-5; W ESTMAN, 2000: 110-111; JONES, 2000). Así, se constata cómo conviven distintas aproximaciones, desde las más tipológicas empleadas para la arquitectura rural -a pesar del debate surgido desde las páginas de Vernacular A rchitecture a finales de los 80 e inicios de los 90-, o las propiamente estratigráficas más empleadas en el caso de la arquitectura monumental e incluso industrial (CLARK, 2000: 17-18; PALMER, N EAVERSON, 1998: 97-103).

En España se han experimentado en el último decenio distintas fórmulas de articular la lectura estratigráfica de al zados. La formulación más empleada hasta el momento es la que, explicitada y normalizada a partir del año 1995, se reconoce esencialmente en las experiencias desarrolladas por algunos equipos de trabajo italianos, como fruto de la reflexión crítica y la experimentación aplicada en numerosos conjuntos monumentales. Sin embargo esto 
no quiere decir que sea la única manera de abordar la lectura estratigráfica. Así por ejemplo, en el caso de la catedral de Santa M aría de Vitoria-Gasteiz se ha experimentado la integración de la lectura estratigráfica de grandes volúmenes guiada por cronotipologías relativas, reinterpretando de forma original estos instrumentos y adaptándolos al análisis de grandes volúmenes (AZKARATE, 2001).

En el caso de Sevilla, en cambio, M. A. Tabales integra en su propuesta de actuación un doble proceso de lectura que combina un análisis estratigráfico con otro que denomina estructural, y en el que se integran estudios de carácter tipológico (aparejos, vanos, enlucidos), con otro tipo de lecturas estrictamente estructural es de carácter no estratigráfico, que inciden en la valoración arqueológica general (TABALES, 2000a: 324-325).

Al margen de otro tipo de consideraciones referidas a las circunstancias en las que se han gestado este tipo de estrategias y procedimientos (derivados de la natural eza de los trabajos de investigación, gestión, etc.), es necesario subrayar que la realidad es muy cal eidoscópica, y que nos encontramos en una fase de verdadera experimentación y puesta a punto de los instrumentos más adecuados.

Otro aspecto que merece la pena ser subrayado es que, desde hace tiempo, se trabaja en la elaboración de un corpus de instrumentos de datación de carácter arqueológico, independiente de otros de carácter formal. A unque se continúa recurriendo con frecuencia a indicadores cronológicos tradicionales, como las fuentes indirectas (y en primer lugar la documentación escrita) o estilísticas (siguiendo una tradición de estudios afirmada, por ejemplo, en la arquitectura al tomedieval), hay un interés creci ente por la potenciación de otro tipo de instrumentos.

Así por ejemplo han empezado a realizarse curvas mensiocronológicas de ladrillos. De hecho, se ha podido ver que en España se empleaban los mismos sistemas de reglamentación de las producciones de materiales constructivos (esencial mente ladrillos y tejas) que en otros países donde se han podido fechar los edificios en función de sus características y dimensiones, como en Ital ia, A lemania o Inglaterra, ya que la tendencia a la reducción de las dimensiones era frecuente en las principales ciudades y centros urbanos (O viedo, Tol edo, Cuenca, Sevilla, Madrid, etc.). Las principales experiencias se han Ilevado a cabo, hasta el momento, en el caso de Valencia, donde X. Martí y sus colaboradoras han propuesto las primeras curvas de referencia (Altarriba $\notin$ alii, 2001).

O tra línea de trabajo que se está desarrollando es la de la elaboración de catál ogos y repertorios territoriales de técnicas constructivas. Ya en el año 1995 se había propues- to la elaboración de este tipo de instrumentos para poder fechar los paramentos y analizar las estructuras productivas vinculadas a la arquitectura, pero hay que reconocer que queda un largo camino por recorrer.

Precisamente el desarrollo de estos instrumentos de datación dependientes de la estructura productiva local 0 regional es un indicador evidente de la madurez y del desarrollo al canzado por la propia disciplina en un determinado territorio. Esto es debido a que, por un lado, requieren de una importante inversi ón de tiempo en la realización de tipologías que podrán aplicarse solamente en ámbitos territoriales reducidos. Las limitaciones observadas en la arquitectura peninsular de época romana, que presenta notables variaciones y diferenciaciones territoriales a pesar de poder ser considerada a priori como una arquitectura tipologicamente homogénea, constituyen un claro reflejo de las limitaciones territoriales de estos instrumentos (BEN DALA, ROLDÁN, 1999).

Pero además es imprescindible decir que, por sí misma, la estratigrafía de alzados no es suficiente para entender la complejidad que presenta el registro arquitectónico. De la misma manera que en una excavación el proceso de investigación no se concluye cuando se ha decodificado la secuencia estratigráfica, tampoco en la arqueología de la arquitectura la formalización de una lectura estratigráfica a través de un diagrama y unas reconstrucciones volumétricas concluyen el proceso lógico de estudio.

En el caso de las excavaciones constituye una fuente de información fundamental el estudio de los objetos mue bles y las informaciones contenidas en el propio sedimento; en la arquitectura tendremos que analizar los materiales y las técnicas constructivas empleadas. La arqueología de la arquitectura cuenta con un utillaje conceptual e instrumental adecuado para analizar los materiales y las formas de construir, estudiando de esta manera los aspectos productivos y social es que se encuentran contenidos en el documento arquitectónico. De hecho las experiencias italianas nos enseñan cómo el estudio de las técnicas constructivas es un instrumento esencial para realizar una historia social de la arquitectura y alcanzar de esta manera el fin último de cualquier actividad arqueológica, hacer Historia (Mannoni, Giannichedda, 1996; Brogiolo, 1996; ManNoni 1994, 1997; BianchI, Francovich, 2002; CAGNANA, 1997)5.

\footnotetext{
${ }_{5}^{5}$ Para el caso peninsular se pueden señalar para el período medieval trabajos como los de Azuar Ruiz, 1995; Gutiérrez González, 1998; Malpica Cuello, 1999; Fernán dez Mier, Quirós CAstillo, 2001.
} 
Por ello es preciso reivindicar la dimensión histórica de la arqueol ogía de la arquitectura, y la necesidad de desarrollar model os interpretativos propios, que superen las lecturas realizadas desde la historia de la arquitectura. En esta tradición historiográfica ha primado una investigación de tradición positivista, muy vinculada a los presupuestos de la Escuela Histórico Cultural, que utiliza categorías de análisis de carácter idealista (por ejemplo las continuas referencias a una historia de estil os: románico, gótico, etc.).

En este contexto cabe hacer una diferencia entre la evolución reciente de la arqueología de época histórica en España respecto a otras tradiciones europeas. En otros países de nuestro entorno los años 70 han supuesto una profunda renovación, tanto desde un punto de vista epistemológico como instrumental, que han llevado a romper con la concepción tradicional entre arqueología como historia del arte, y a replantear la disciplina en el marco de la historia de la cultura material (CARAN DINI, 1984).

U na de las principales consecuencias de esta renovación ha consistido en la desmonumental ización de la práctica arqueológica, y en la introducción de la historia de los grupos dominados en el discurso arqueológico (p.e. Chapelot, Fossier, 1980; AA. VV., 1980; Ferrando CABOnA, CRUSI, 1988). Por toda una serie de complejas razones, no parece que en España esta renovación haya impregnado de forma general la práctica de la arqueología de época medieval, e histórica en general, e incluso se observan diferencias notables entre las instituciones acadé micas, más tradicionales, y las empresas de arqueología, más avanzadas. Los recientes estudios sobre los asentamientos campesinos altomedievales no dejan de ser un óptimo ejemplo sobre la necesidad de ampliar el campo de estudios y, sobre todo, de plantear un programa de investigación que permita revalorar la arqueología de la arquitectura como disciplina arqueológica (VIGIL Escalera, 2000).

\section{LA LEGISLACIÓN Y LA ADMINISTRACIÓN}

La ley de Patrimonio H istórico Español del año 1985 define el patrimonio arqueológico como los bienes muebles e inmuebles "susceptibles de ser estudiados con metodología arqueológica" (art. 40.1). Desde este punto de vista, la legislación ampara la práctica de la arqueología de la arquitectura, en cuanto recurre a una metodología arqueológica. Este principio básico - reconocido y reflejado por todas las legislaciones autonómicas (QUeROL, MARTín EZ DíAZ, 1996: 129 ss.)- entra en cambio en contradicción con el tipo de intervenciones arqueológicas reconocidas por la legislación, ya que se reducen a la exca- vación y a la prospección. Se crea de esta manera una contradicción, en cuanto queda en manos de la sensibilidad de los administradores la regulación y la oportunidad o la necesidad de llevar a cabo este tipo de estudios.

Solamente en las propuestas normativas más recientes, se da espacio también a las lecturas arqueológicas de alzados, y se propone la necesidad de realizarlas bajo autorización específica.

En la actualidad, las únicas normativas que recogen en su articulado la necesidad de intervenir en las restauraciones a través de anál isis arqueol ógicas de alzado se reducen a las municipales, que se han demostrado como las de mayor dinamismo y flexibilidad. Entre estas es necesario señalar por su carácter pionero la del ayuntamiento de M álaga, que establece una tasa en aquellos casos en los que sea necesario intervenir en el subsuel o 0 en "instalaciones susceptibles de aplicación de arqueol ogía de la arquitectura o industrial".

Asimismo, el Plan Territorial Sectorial del Patrimonio Cultural del País Vasco, será una de las primeras normativas legislativas de ámbito autonómico que recoja de forma específica las intervenciones en este ámbito concreto de estudios. La importancia de este documento reside en el hecho de que se trata de un texto jurídico destinado a coordinar las leyes de Patrimonio Cultural y de Ordenación del Territorio del año 1990, por lo que se puede convertir potencialmente en un instrumento de gran utilidad que permita incorporar la arqueol ogía de la arquitectura en el ámbito de la intervención sobre el patrimonio edificado y en la planificación urbanística.

Así pues, debemos constatar que existe un vacío legal en lo que se refiere a la práctica de la arqueología de la arquitectura, lo que beneficia por un lado su aplicación por la inexistencia de trabas burocráticas, pero a la vez impide su generalización.

\section{ARQUEOLOGÍA DE LA ARQUITECTURA Y RESTAURACIÓN} Un último aspecto que es necesario abordar es el de la aplicación de la arqueol ogía de la arquitectura en el ámbito de las intervenciones de rehabilitación y restauración del patrimonio edificado, lo que nos Ileva a ocuparnos directamente de la relación entre arqueólogos, arquitectos, arquitectos restauradores, restauradores y profesionales implicados en la restauración de monumentos.

Desde hace ya al gunos años, el debate en I tal ia versa esencial mente en torno a cómo los arquitectos emplean los instrumentos estratigráficos, cómo se convierten en arqueólogos, y cómo los mismos arqueólogos adaptan sus 
recursos para adecuarse a las necesidades impuestas por la propia práctica restauradora.

A ctual mente este debate no tiene ecos en la situación de la Península Ibérica, ya que nos encontramos en un momento de experimentación y evaluación del potencial de esta disciplina en el ámbito de la rehabilitación y restauración arquitectónica. Las experiencias no son aún muy numerosas, y aunque se presentan muy prometedoras, evidentemente no están exentas de al gunos problemas.

El primero de ellos, es el de la tendencia, tal como sucede en I talia, a la simplificación einstrumentalización de la disciplina. Así, al gunos autores identifican la arqueología de la arquitectura con un "método", y se define como "una herramienta para conocer y determinar las relaciones temporales que se producen entre los materiales de un edificio" (LATORRE, 1996: 103, 115; LeCANdA Esteban, 2000: 341). Es cierto que son precisamente los arquitectos restauradores los que han dado un impulso notable para que se experimentase la potencialidad de los instrumentos arqueológicos en el ámbito de la rehabilitación arquitectónica. Sin embargo, solamente a partir de un desarrollo maduro de la arqueología de la arquitectura se podrá superar la tan arraigada identificación entre arqueología y estratigrafía, entre arqueólogo y técnico-que-nosproporciona informaciones (BROGIOLO, 1997). La investigación histórica y arqueológica no es un convidado de piedra más, sino que constituye la fuente y el instrumento capaz de dotar de significados y val ores a una arquitectura que de esta manera permite su social ización y, por lo tanto, su preservación.

La complejidad y responsabilidad que plantea la intervención en el patrimonio edificado es tal, que no se puede delegar en un solo profesional, en una sola disciplina, la toma de decisiones sobre cómo intervenir. $\mathrm{H}$ ace ya unos años Carandini planteaba cómo más al lá de la estética se encuentra la ética del contexto, y estees un principio que no puede englobarse en genéricos valores históricos de los edificios. Por ello no parece razonable que la arqueología de la arquitectura se reduzca a un instrumento que ofrezca una lectura diacrónica de los edificios que convalide y supere otras formas de lectura aplicadas hasta el momento.

En la actual idad se percibe una notable disparidad en los criterios de intervención, y frente a la posición más extrema - representada en España por la plena integración en el proyecto de los arqueólogos, como en el caso de la intervención en la catedral de Vitoria-Gasteiz (AzKARATE, 2001)-, sigue siendo frecuente que el papel de la arqueología se limite a la fase previa de intervenciones cognoscitivas.
Por otro lado, parece claro que la arqueología de la arquitectura ofrece un marco conceptual nuevo para superar la situación tradicional de los últimos decenios en el que los estudios de carácter histórico se convertían en un mero apéndice de las investigaciones real izadas de forma previa a la rehabilitación arquitectónica, con frecuencia subordinados a la interpretación gl obal real izada sol amente por los arquitectos (TAGLIABUE, 1993; DogLIONI, 1997).

Es importante subrayar, no obstante, cómo se han dado pasos de gigante para lograr construir estrategias integrales de intervención en el patrimonio edificado (Latorre, 1995; González Moreno, N avarro, 1999) absolutamente necesarias en la situación actual, aunque siguen persistiendo las incomprensiones y los problemas en la práctica cotidiana.

No obstante, hasta el momento el empleo de la lectura estratigráfica parece mantenerse - salvo excepciones puntual es (MILETO, 2000)- como un instrumento propio y exclusivo de arqueólogos y de los restauradores (CORTAZAR, 2000), y aún estamos lejos de una socialización de la estratigrafia entre distintos profesionales, paraIela a la que ha tenido lugar en I talia (BRogIOLo, 2002).

Estas dificultades en la difusión y recepción crítica de la arqueología de la arquitectura se deben, esencialmente, a la carencia de una formación reglada, tanto en lo que se refiere a los arquitectos como a los arqueólogos, e incluso por la carencia de una literatura en castellano sobre la materia.

A este propósito se han realizado en los últimos años cursos de postgrado y masters dirigidos a arquitectos restauradores en los que se han impartido nociones sobre la arqueología de la arquitectura, y al que han participado tanto arqueól ogos como arquitectos sensibles al empleo de este bagaje instrumental. A simismo, se debe señalar cómo en la actual idad hay dos arqueól ogos impartiendo clases de arqueología en las escuel as de arquitectura de M adrid y de Sevilla (F. Vela Cossío, M. A. Tabales).

Estas mismas carencias se observan asimismo en el caso de la formación de los arqueólogos en España. Según nuestras noticias, solamente en la Universidad del País Vasco existe una asignatura dedicada a esta materia desde el plan de estudios del año 1993 (denominada entonces "A nálisis estratigráfico de estructuras en alzado", y desde el año 1998 "A rqueología de la A rquitectura"). A simismo, se imparten estos contenidos en algunos cursos de doctorado, como los promovidos en los últimos años por $A$. M al pica en la U niversi dad de Granada.

Otro aspecto que hay que resaltar es que, hasta el momento, se ha recurrido a la arqueología de la arquitec- 
tura esencialmente en el marco de los conjuntos monumentales y edificios de una cierta entidad, esencialmente en el ámbito de la gestión. Estas circunstancias han permitido encarar estos estudios con medios económicos y humanos suficientes como para que se formasen algunos equipos multidisciplinares que pudiesen recurrir de forma general izada a sistemas complejos de documentación gráfica (en particular el recurso a la restituciones fotogramé tricas), o que se desarrollasen complejos programas arqueométricos (como por ejemplo las dataciones arqueométricas real izadas en la arquitectura al tomedieval peninsular son dos ejemplos significativos: A LONSO Mathías e alii, 1997; CAballero Zoreda \& alii, 1997; Rodríguez Trobajo et alii, 1998).

Creemos, sin embargo, que uno de los retos que tendrá que abordar la arqueol ogía de la arquitectura peninsular en los próximos años será precisamente el de ampliar sus ámbitos de trabajo a otros registros arquitectónicos, y desarrollar nuevas estrategias más ágiles y flexibles para intervenir también en edificios de otra natural eza.

A pesar de que los debates y la atención por las intervenciones de restauración y rehabilitación se concentran esencial mente en el marco de la arquitectura monumental, donde existe un mayor control, es precisamente en la arquitectura rural y residencial en los cascos históricos, el patrimonio edificado más frágil y más sujeto a transformaciones radical es, donde se produce una erosión más importante de estos val ores y donde es preciso desarrol lar instrumentos y estrategias adecuadas.

Es preciso que surja, al lado de la investigación de alto nivel, una serie de procedimientos y estrategias que puedan generar una "arqueología de intervención" en el ámbito de la arquitectura. De hecho, aún no se ha explorado de forma adecuada la aplicación de la arqueología de la arquitectura como instrumento de eval uación y programación en el ámbito de la urbanística y de la ordenación del territorio, ya que las experiencias son aún muy reducidas (BrogIolo, 1988). U rge, por lo tanto, que se potencie una investigación específica dirigida a desarrollar rutinas de investigación arqueológica que permitan su difusión y general ización, garantizando el rigor metodológico, pero a su vez haciendo más flexibles los sistemas de registro y análisis.

Por último puede merecer la pena señal ar asimismo la necesidad de trabajar de forma más intensa en el desarrollo de una arqueometría aplicada a la arqueología de la arquitectura. Existen laboratorios especial izados que cuentan con una buena experiencia dirigida al estudio de la procedencia de los materiales constructivos, como por ejemplo en el caso de los materiales ornamentales de época romana (Álvarez Pérez, Mayer, RodÀ, 1992), o en la definición de fases constructivas a partir de las diferencias de morteros y argamasas (p.e. Bertrán, Fernández, 1990), por señalar solamente dos cuestiones específicas.

Sin embargo, la rutina de investigación sigue siendo, desde nuestro punto de vista, aún demasiado lineal, echándose en falta una mayor interacción entre los distintos investigadores que permita abordar la realización de programas arqueométricos más maduros. N umerosas técnicas constructivas han desaparecido con la industrialización y la extinción de conocimientos tecnológicos transmitidos de forma empírica por el artesanado tradicional. La arqueología de la arquitectura tiene mucho que decir también en este campo, pero para ello debe implicarse de forma más activa en investigaciones arqueométricas más globales y amplias que las realizadas hasta el momento.

\section{CONCLUSIONES}

Somos conscientes de que en la actualidad la arqueología de la arquitectura puede ser considerada como una disciplina arqueológica que está "de moda", en cuanto genera un notable interés por parte de los propios arqueólogos, pero también en otras disciplinas más o menos afines, empeñadas en el estudio y gestión del patrimonio edificado. Seguramente los aspectos más atractivos de esta disciplina se basan en la posibilidad de ampliar el ámbito de trabajo de la misma disciplina arqueológica hacia un campo hasta el momento dominado por los instrumentos y el bagaje conceptual de la historia del arte y de la arquitectura, y por la capacidad de dar respuesta a una demanda social, la del estudio y valorización de la arquitectura como documento histórico y como significante social.

La arqueología española ha conocido en los últimos decenios numerosas "arqueol ogías" o innovaciones resultado de la "importación" de experiencias y de reflexiones realizadas en el marco europeo. Baste recordar la introducción en los años ochenta del Ilamado "método Harris" de registro, o de la en su día denominada "arqueol ogía espacial". Todas estas arqueologías han precisado de un largo proceso de decantación y de experimentación crítica gracias a la cual se ha logrado dotar a la arqueología de un lenguaje de carácter estratigráfico, o ha surgido una arqueología del paisaje tan sólida como la actual, capaz de integrar la dimensión científica y aplicada del estudio arqueológico del paisaje (CRIADO, 1999).

Este es precisamente el camino que se abre ahora a la arqueol ogía de la arquitectura, teniendo en cuenta el carác- 
ter bidimensional que tendrá que mantener este tipo de estudios desde su compromiso social en la gestión y puesta en val or del patrimonio edificado, y desde la necesidad de construir una historia social de la arquitectura.

\section{Bibliografía}

AA. VV., 1980, Per una storia delle dimore rurali, A rcheol ogia M edievale, VII, pp. 7-436.

AA. VV., 1996, International updating course on the role of preparatory architectural investigation in the restoration of historical buildings, Leuven.

AA. VV., 1997, El monument, document, Quaderns científics i tènnics, 9, Diputació de Barcelona, Barcel ona, pp. 351-367.

ACIEN M., 1999, La torre del homenaje de la Alcazaba de Málaga. Secuencia, estratigrafía, medición e interpretación, en A rqueología del M onumento. A ctas de Ios III encuentros sobre A rqueología y Patrimonio, Salobreña, pp. 173-203.

Almagro A., Caballero L., Cámara L., Latorre P., 1992, Investigación y restauración de la iglesia visigoda de Santa Lucía del Trampal, Alcuescar (Cáceres), en A ctuacions en el patrimoni edificat: la restauració de l'arquitectura del s segles IX i X (Investigació històrica i disseny arquitectònic), Quaderns científics i técnics, 4, Barcelona, pp. 95-110.

Alonso Mathías F., Caballero Zoreda L., Rodríguez TROBAJO E., 1997, Cronología constructiva de la iglesia mozárabe de $S$. Baudelio de Berlanga (Soria): primeros resultados de dendrocronol ogía y carbono-14, B ol etín de A rqueología M edieval, 11, pp. 249-263.

Altarriba M., Guillén C., Guzmán R., Rojo N., Martí J., 2001, U na propuesta de curva mensiocronológica latericia para la ciudad de Valencia, en A ctas de V Congreso de A rqueol ogía M edieval Española, Valladolid, pp. 235-261.

Álvarez Pérez A., Mayer M., Rodà I., 1992, El análisis de materiales Iapídeos, en RODA I., Ciencias, metodologías y ténicas aplicadas a la arqueología, Barcelona, pp. 247-260.

APARICIO BASTARDO J. A., 1990, Análisis de fases constructivas: aproximación al caso burgalés, en II J ornadas B urgalesas de $H$ istoria. B urgos en la A Ita Edad M edia, Burgos, pp. 445-456.

ARCE I., 1999, El análisis estratigráfico y la intervención sobre edificios históricos: el caso del Palacio Omeya de A mman, en Tratado de rehabilitación, tomo 2: Metodol ogía de la restauración y de la rehabilitación, Madrid, pp. 237-250.

AZKARATE GARAI-OLAUN A., 1995, A portaciones al debate sobre arquitectura prerrománica peninsular: la iglesia de San Román de Tobillas (Álava), A rchivo Español de A rqueología, 68, pp. 189-214.

AzKarate Garai-Olaun A., 2001, Análisis de la evolución históricoconstructiva de la catedral de Santa M aría de Vitoria-Gasteiz (A plicación de la 'A rqueología de la Arquitectura' a un modelo complejo), en A ctas de V Congreso de A rqueología M edieval Española, Valladolid, 1999, Volumen 1, pp. 177-190.

AzKarate Garai-Olaun A., 2002, Intereses cognoscitivos y praxis social en A rqueología de la A rquitectura. Experiencias desde la U niversidad del País Vasco, A rqueología de la A rquitectura, 1, pp. 55-71.

Azuar R., Bevía M., Bevía P., López J. A., Menéndez J. L., ORTEGA J. R., SÁN CHEZ M. D., 2000, A rqueología de la arquitectura: excavación de las bóvedas de la iglesia gótica de Santa M aría de A licante (siglo $X V$ ), en $A$ ctas de $V$ Congreso de A rqueología M edieval Española, Valladolid, vol.1, pp. 351-359.

AZUAR RUIZ R., 1995, Las técnicas constructivas en al-Andalus. El origen de la sillería y del hormigón de tapial, en $V$ Semana de Estudios M edievales de Nájera, Logroño, pp. 125-142.

BEDAL K., 1993, Historische Hausforschung: Eine Einführung in A rbeitsweise, $B$ egriffe und Literatur, F ränkisches $F$ rèlichtmuseum, Bad W indsheim.

BEN DALA GALÁN M., 1992, Materiales de construcción romano: peculiaridades de H ispania, en RoDA I., Ciencias, metodologías y ténicas aplicadas a la arqueología, Barcel ona, pp. 215-226.

Bendala Galán M., Rico C., Roldán Gómez L. (eds.), 1999, El ladrillo y sus derivados en la época romana, Monografías de Arquitectura romana, 4, Madrid.
Bendala Galán M., Roldán Gómez L., 1999, El cambio tecnológico en la arquitectura hispanorromana: perduración, novedades y peculiaridades, en II Congreso de A rqueol ogía Peninsular, tomo IV: A rqueol ogía romana y medie val, Madrid, pp. 103-115.

BERTRÁN R., FERNÁN DEZ J ., 1990, Estudi dels morters medievals, en La vida medieval a les dues vessants de Pirineu. $1 \mathrm{r}$ i $2^{a} \stackrel{\text { curs d}}{ }$ d'arqueologia d'A ndorra, Andorra, pp. 169-172.

BIANCHI G., FRAN COVICH R., 2002, L'archeologia dell'architettura come archeologia, A rqueol ogía de la A rquitectura, 1, pp. 101-111.

B LAN CO R OTEA R., 2000, Las construcciones históricas desde una perspectiva arqueológica: Lectura de paramentos, en BORES GamUN DI F., FERNÁN DEZ Salas J., Huerta Fernández S., Rabasa Díaz E. (eds.), A ctas de Segundo Congreso $\mathrm{N}$ acional de H istoria de la Construcción (A Coruña, 22-24 octubre 1998), Madrid, pp. 49-56.

BLANCO ROTEA R., e.p., The study of medieval architecture from an archaeological perspective, en XIV th Congress of the U I. I.S.P.P., Liège, B elgique, 2.8 sept. 2001, British Archaeological Reports, International Series, Oxford.

BRogiolo G. P., 1988, A rcheologia del'edilizia storica, Como.

BROGIOLO G. P., 1995, A rqueología estratigráfica y restauración, Informes de la construcción, vol. 46, n. 435, pp. 31-35.

Brogiolo G. P., 1996, Prospettive per l'archeologia dell'architettura, A rcheologia dell'A rchitettura, 1, pp. 11-15.

BROGIOLO G. P., 1997, Dall'analisi stratigrafica degli elevati all'A rcheologia dell'architettura, A rcheologia del'A rchitettura, 2, pp. 181-184.

BRoGIOLO G. P., 2002, L'archeologia dell'architettura in Italia nell'ultimo quinquennio (1997-2001), A rqueología de la A rquitectura, 1, pp. 19-26.

Caballero L., Cámara L., 1995, Un caso de lectura de paramentos y argumentación científica. San Pedro el Viejo de Arlanza, Burgos-España, Informes de la Construcción, vol. 46, n. 435, pp. 79-90.

CABAllero Zoreda L., 1987, El método arqueológico en la comprensión del edificio (sustrato y estructura), en Curso de M ecánica y Tecnología de los Edificios A ntiguos, COAM, Madrid, pp. 13-58.

CABAllero ZoRedA L., 1994-1995, Un canal de transmisión de lo clásico a la alta Edad M edia española. A rquitectura y escultura de influjo omeya en la Península Ibérica entre mediados del siglo VIII e inicios del siglo X, A IQantara, pp. 25 y 26, 321-348 y 107-124.

CABAllero Zoreda L., 1997, En torno a algunas experiencias de lectura arqueológica de edificios, Quaderns Científics i Téćnics, 9, pp. 307-324.

Caballero Zoreda L., Escribano Velasco C. (eds.), 1996, A rqueol ogía de la A rquitectura. El método arqueol ógico aplicado al proceso de estudio y de intervención en edificios históricos, J unta de Castilla y León, Valladolid.

Caballero Zoreda L., Fernández Mier M., 1997, Análisis arqueológico de construcciones históricas en España. Estado de la cuestión, A rcheologia del'architetura, 2, pp. 147-158.

Caballero Zoreda L., LATORRe P., 1995, Leer el documento construido, Informes de la Construcción, 435, pp. 3-100.

Caballero Zoreda L., Sanz J., Rodríguez Trobajo E., A LON SO M ATHÍAS F., 1997, San Pedro de la N ave (Zamora). Excavación arqueológica del solar primitivo de la iglesia y análisis por dendrocronología y carbono-14 de su viga, A nuario Instituto de Estudios Z amoranos Florián de Ocampo, 1997, pp. 43-57.

CAgn An A A., 1997, La transizione al Medioevo attraverso la storia delle tecniche murarie: dall'analisi di un territorio a un problema sovraregionale, en I Congresso $\mathrm{N}$ azionale di A rcheologia M edievale (Pisa, 29-31 maggio 1997), Firenze, pp. 445-448.

CAÑ AVATE J. (ed.), 2001, A rquitectura y A rqueol ogía M edieval, Granada.

CARANDIN I A., 1984, A rqueología y cultura material, Barcelona.

CARANDINI A., 1998, H istorias en la tierra, Barcelona.

Castillo Armenteros J. C., Castillo Armenteros J. L., Marín García M. M., Pérez M artínez M. C., 1999, La arqueología de apoyo a la restauración: teoría o realidad. El caso de las fortificaciones giennenses, en A rqueología de M onumento. A ctas de los III encuentros sobre A rqueología y Patrimonio, Sal obreña, pp. 205-230. 
Chapelot J., Fossier R., 1980, Le village et la maison au M oyen Â ge, Paris.

CLARK K ., 2000, Architect's specification: building analysis and conservation, en Rosk AmS S. (eds.), Interpreting Stratigraphy. Site evaluation, recording procedures and stratigraphic analysis. Papers presented to the Interpreing Stratigraphy Conferences 1993-1997, British Archaeological Reports, International Series 910, Oxford, pp. 17-24.

COBOS GUERRA F., 2000, El Plan Director de restauración del Castillo de la M ota. M etodología de estudio e intervención, en J. RIVERA (coord.), Congreso Internacional sobre restauración de ladrillo, Valladolid, pp. 99-112

Coll Conesa J., Huélamo Gabaldón J. M., Solias A rís J . M., 1992, L'edifici de la Inquisició de Cuenca. Avanç a la metodologia desenvolupada al seu estudi arqueo-arquitectònic, en BRócolI I. G., SOSPEDRA R. (eds.), H arris M atrix. Sistemes de R egestre en A rqueología, Lleida, vol. 2, pp. 77130.

CORTAZAR M ., 2000, Estudio de correspondencia de policromías del apostoIado del Pórtico Viejo de San Pedro (Vitoria), A kobe R estauración y conservación de bienes culturales, 1, pp. 8-11.

CRIADO BoAdo F., 1996, El Futuro de la Arqueologia, ¿La Arqueologia Del Futuro?, Trabajos de Prehistoria, 53 (1), pp. 15-35.

CRIAdo Boado F., 1999, Del Terreno al Espacio: Planteamientos y Perspectivas para la Arqueología del Paisaje, CAPA 6, Santiago de Compostela.

DAVIES M., 1993, The application of the $H$ arris Matrix to the recording of standing structures, en HARRIS E. C., Brown III M.R., Brown G.J. (eds.), Practices of archaeological stratigraphy, London, pp. 167-180.

D OGLIONI F., 1997, Stratigrafia e restauro. Tra conoscenza e conservazione del'architetura, Trieste.

DuprÉ RAVEnTÓS X., 1994, L'arc romà de Berà (Hispania Citerior), Monografías de la Escuela Española de H istoria y A rqueología en Roma, 20, Roma.

DUPRÉ R AVENTÓS X ., 1997, Prólogo a la edición española, en CARANDINI A., H istorias en la Tierra. M anual de excavación arqueológica, Barcelona, pp. VIIXVIII.

Escribano Velasco C., 1999, La iglesia de Santa María la Mayor (Villanueva de los Infantes, Valladolid). Lectura estratigráfica de un nuevo templo prerrománico, en II Congreso de A rqueología Peninsular, tomo IV: A rqueol ogía romana y M edieval, Madrid, pp. 457-474.

ESQUIEU Y., 1997, L'archeologie du bâti en France, A rcheologia del'A rchitettura, 2, pp. 133-140.

Estrade Amézaga E., Arostegui Irastorza M. J. (dirs.), 2000, A vance de Plan teritorial Sectorial de Patrimonio Cultural Vasco, Gobierno Vasco, Vitoria-Gasteiz

FEIJ 00 S., 1997, Aspectos constructivos del Puente Romano de Mérida, en M érida Excavaciones A rqueológicas 1997, Mérida, pp. 321-337.

Fernández Mier M., Quirós CAstillo J. A., 2001, La evolución de las técnicas constructivas en Asturias en la Edad M edia, en $A$ ctas del $V$ C ongreso de A rqueología M edieval E spañola, Valladolid, vol.1, pp. 371-382.

Ferrando Cabona I., Crusi E., 1988, Storia del'insediamento in L unigiana. A Ita Valle A ulella, Génova.

Ferrando Cabona I., Mannoni T., Pagella R., 1989, Cronotipologia, A rcheol ogia M edievale, XVI, pp. 647-661.

Fran COVICH R., PARENTI R. (eds.), 1988, A rcheologia e R estauro dé monumenti. $I^{\circ}$ ciclo di lezioni sulla ricerca applicata in archeol ogia. Certosa di Pontignano (Siena), 28 settembre 10 ottobre 1987, Firenze.

Galindo Torres J., Mauri Martí A., Rovira Tovella R., 1994, Les tècniques constructives del castell de Gelida, en IV Congreso de A rqueología M edieval Española, "Sociedades en transición", Alicante, vol. 2, pp. 387-392.

García de CASTRO VALDÉs C., 1997, Un enfoque arqueológico de la arquitectura al tomedieval asturiana, en HEVIA BLANCO J. (comp.), La intervención en la A rquitectura prerrománica asturiana. J ornadas sobre intervención en $\mathrm{e}$ Patrimonio A rquitectónico asturiano, O viedo, pp. 149-165.

GONZÁLEZ MORENO-N AVARRO A., 1999, La restauración objetiva (método SCCM de restauración monumental), M emoria SPA L 1993-1998, 1, Barcelona.
GREN VILLE J ., 1997, M edieval H ousing, Leicester U niversity Press, London$N$ ew York

GuTIÉRREZ GonzÁLEZ J. A., 1998, La construcción militar medieval. Tecnología y sociedad, en Técnicas agrícolas, industriais e constructivas na idade media, Vigo, pp. 277-336.

Jiménez M ARTín A., 1989, La Puerta de Sevilla en Carmona, Sevilla.

J ONES M., 2000, The stratigraphic examination of Standing Buildings: problems and solutions, en Rosk AMS S. (ed.), Interpreing Stratigraphy. Site evaluation, recording procedures and stratigraphic analysis. Papers presented to the Interpreting Stratigraphy Conferences 1993-1997, British Archaeological Reports, International Series 910, 0xford, pp. 125-129.

J OURN OT F., 1999, Archéologie du Bâti, en La construction en pierre, Paris, pp. 133-163.

K IMPEL D., 1995, La actividad constructiva en la Edad Media: estructura y evolución, en CASSANELLI R. (ed.), Talleres de A rquitectura en la Edad M edia, Barcelona, pp. 11-50.

LAMBOGLIA N ., 1958, Opus certum, Rivista di Studi Liguri, XXIV (1-2), pp. 158-170.

LATORRE GonzÁLEZ-M ORO P., 1996, La arqueología de la arquitectura. Consecuencias metodológicas de su aplicación al proyecto de restauración, en L. Caballero Zoreda, C. Escribano Velasco (eds), A ctas. A rqueología de la A rquitectura: El método arqueol ógico aplicado al proceso de estudio y de intervención en edificios históricos, Sal amanca, pp. 103-121.

LATORRE P., 1995, Análisis arqueológico de los paramentos del faro romano Ilamado Torre de H ércules. La Coruña-España, Informes de la Construcción, vol. 46, n. 435, pp. 37-46.

LATORRE P., CABALLER O L., 1995, La importancia del análisis estratigráfico de las construcciones históricas en el debate sobre la restauración monumental, Informes de la Construcción, vol. 46, n. 435, pp. 5-18.

LECAN DA ESTEBAN ]. A., 2000, El análi isis arqueológico de la iglesia de San Salvador de Escaño: resolución de problemas arquitectónicos y aproximación histórica al primer románico burgal és, en $A$ ctas del $V$ Congreso de A rqueol ogía M edieval Española, Valladolid, vol.1, pp. 341-349.

LÓPEZ MULLOR A., 1994, Arqueología y función social. Alguna teoría y unos cuantos ejemplos, A rqueología y territorio medieval, 1, pp. 51-63.

LÓPEZ MULLOR A., 1999, D os estudios recientes de arqueología del patrimonio edificado, en XX V Congreso $\mathrm{N}$ acional de A rqueol ogía, Val encia, pp. 161177.

LóPEZ M U LLOR A, 2002, Veinte años después, A rqueol ogía de la A rquitectura 1, pp. 159-174.

Magalhaes Ramalho M. M. B., 1996, A archeologia na intervençao dos edificios históricos ou a Arqueologia da arquitectura, A I-M adan, 5, pp. 50-56.

Maldonado Ramos L., Vela Cossío F., 1998, De A rquitectura y A rqueol ogía, Madrid.

Mald OnAdo Ramos L., Vela Cossío F., 1999, Estrategia y metodología de la intervención en edificios históricos. Una perspectiva desde la arquitectura y la arqueología, en Tratado de rehabilitación, tomo 2: M etodología de la restauración y de la rehabilitación, Madrid, pp. 219-227.

MaLONEY S. J., RingBOM M., 2000, 14C dating of mortars at Torre de Palma, Portugal, en V Reunió d'A rqueología Cristiana H ispánica, Cartagena (1998), Barcelona, pp. 151-155.

Malpica Cuello A. (ed.), 1999, A rqueología de monumento: A ctas de los Tercer os E ncuentros de A rqueol ogía y Patrimonio, Sal obreña, del 13 al 16 de octubre de 1992, Salobreña.

M ALPICA CUELLO A..1999, Las técnicas constructivas en Al-Andalus. Un debate entre la arqueol ogía y la arquitectura, en Técnicas agrícol as, industriais e constructivas na idade media, Vigo, pp. 229-276.

MAN N ONI T., 1990, Archeologia dell'architettura, N otiziario di A rcheologia M edievale, 54, pp. 28-29

M AN N ONI T., 1994, Caratteri costruttivi del'edilizia storica. Venticinque anni di A rcheol ogia globale 3, Genova.

MANNONI T., 1997, II problema complesso delle murature storiche in pietra 1. Cultura materiale e cronotipologia, A rcheologia dell'A rchitettura, 2, pp. 15-24. 
M AN N ON I T., 1998, A nalisi archeologiche degli edifici con strutture portanti non visibili, A rcheologia dell'A rchitettura, 3, pp. 81-85.

Mannoni T., GianNiCHEDDA E., 1996, A rcheologia dela produzione, Turín.

M ATeos Cruz P., 1999, La basílica de Santa. Eulalia de M érida: Arqueología y urbanismo, A nejos de A rchivo E spañol de A rqueología, 19, Madrid.

Men ÉndeZ GRANDA A., 2000, El puente romano de Colloto (Asturias). Intervención arqueológica y lectura de paramentos, en A ctas do 3 0 C ongresso de A rqueol ogía Peninsular, vol. VI: A rqueología da antiguidade na Península I bérica, Porto, pp. 293-303.

MILETO C., 2000, Algunas reflexiones sob-ela A nálisis Estratigráfico de fábrica, Loggia, A rquitectura y Restauración, n. 9, pp. 80-93.

MORRISS R. K., 2000, The archaeol ogy of $B$ uildings, Stroud.

PALMER M., N EAVERSON P., 1998, Industrial archaeology: principles and practice, R outledge, Londres.

PARENTI R ., 1994, La aplicación del método estratigráfico para el análisis de monumentos, en Patrimonio y ciudad. R eflexión sobre centros H istóricos, Córdoba, pp. 58-66.

PARENTI R ., 1995, H istoria, importancia y aplicaciones del método de lectura de paramentos, Informes de la Construcción, vol.46, pp. 19-29.

PARENTI R ., 1996, Una visión general de la A rqueología de la A rquitectura, en Caballero Zoreda L., Escribano Velasco C. (eds), A ctas. A rqueología de la A rquitectura: El método arqueol ógico aplicado al proceso de estudio y de intervención en edificios históricos, Salamanca, pp. 13-21.

PARENTI R ., 1997, La edilicia histórica, la estratigrafía mural y la trascripción de las fuentes documentales, Cuadernos de la A Ihambra, 29-30 (1993-1994), pp. 57-76.

PARENTI R., 2001, Arqueología de la Arquitectura, en Francovich R., M AN ACORDA D. (eds.), D iccionario de A rqueología, Barcelona, pp. 41-45.

Pringent D., HUNOT J. Y., 2000, Archéologie et monuments historiques, D ossiers d'A rcheol ogie, 250, pp. 72-75

Querol M. A., Martínez Díaz B., 1996, La gestión del Patrimonio A rqueológico en E spaña, Madrid.

Quirós CASTILLO J. A., 1994, Contribución al estudio de la arqueología de la arquitectura, A rqueol ogía y territorio medieval, 1, Jaén, pp. 141-158.

Quirós CAstillo J. A., 1996, Indicadores cronológicos de ámbito local: cronotipología y mensiocronología, en Caballero Zoreda L., EsCRIBANo Velasco C. (eds), A ctas. A rqueología de la A rquitectura: El método arqueológico aplicado al proceso de estudio y de intervención en edificios históricos, Salamanca, pp. 179-187.

QUIRÓS CASTILLO J . A., 1998, La sillería y las técnicas constructivas medie vales: historia social y técnica de la producción arquitectónica, A rcheologia M edievale, XXV, pp. 235-246.

QUIRÓS CASTILLO J. A., 2000, La sillería en la arquitectura al tomedieval en la Península Ibérica y en el M editerráneo Occidental, en A ctas del V Congreso de A rqueología M edieval Española, Valladolid, vol.1, pp. 281-291.

RODA I., 1994, Los materiales de construcción en Hispania, en A ctas X IV Congreso Internacional de A rqueología Clásica: La ciudad en el mundo romano, Tarragona, pp. 323-334.

Rodríguez Trobajo E., Alonso Mathías F., Caballero ZOREDA L., 1998, Datación de una viga de la iglesia de San Pedro de la $\mathrm{N}$ ave (Zamora), A rchivo E spañol de A rqueología, 71, pp. 283-294.

ROSK AMS S. (ed.), 2000, Interpreting Stratigraphy. Site evaluation, recording procedures and stratigraphic analysis. Papers presented to the Interpreting Stratigraphy Conferences 1993-1997, British Archaeological Reports, International Series 910, 0 xford.

ROUGER E., 1998, Du principe d'analyse-stratigraphique à l'archéologie d'élévation: réflexion et méthode, A rchéologie M édiévale, 28, pp. 161-172.

SÁN CHEZ J ., 1998, La A rqueología de la A rquitectura. A plicación de nuevos modelos de análisis a estructuras de la Alta Andalucía en época Ibérica, Trabajos de Prehistoria, 55 (2), pp. 89-109.

SÁn Chez Zufiaurre L., Domín guez Beltrán de Heredia I. C., 2000, De la teoría a la práctica: aplicación de lectura estratigráfica a la Torre de Orgaz en Fontecha (Álava), en A ctas del V Congreso de A rqueología M edieval Española, Valladolid, vol.1, pp. 213-220.
Souto LASALA J., 1986, Sobre el papel del arqueólogo medievalista en las obras de restauración de monumentos arquitectónicos, en Actas del primer Congreso de A rqueol ogía M edieval Española, H uesca, vol. 2, pp. 89-102.

TABALES R ODRÍGUEZ M. A., 1999, Análisis arqueológico de paramentos. A plicación es en el patrimonio edificado sevillano, SPA L 6 (1997), pp. 263295

TABALES R OdRíGUEZ M. A., 2000a, Análisis arqueológico de paramentos. A I gunas reflexiones referentes a las últimas actividades sobre inmuebles históricos en la ciudad de Sevilla, en A ctas del $V$ Congreso de A rqueología M edieval Española, Valladolid, vol.1, pp. 319-328.

TABALES R OdRíGUEZ M. A., 2000b, La Arqueología M edieval y sus aportaciones al Conocimiento de las Técnicas Constructivas, en Graciano A. (coord.), La Técnica de la A rquitectura M edieval, Sevilla, pp. 35-74.

TAGLIABUE R ., 1993, A rchitetto e A rcheologo. Confronto fra campi disciplinari, Quaderni del Dipartimento di Conservazione e Storia dell'Architettura, Facoltà di Architettura, Politecnico di Milano n. 5, Milán.

TUDANCA CASERO J. M., 2001, Monumento y yacimiento arqueológico: definición, aproximación y estudio, en TUdANCA CASERo J. M. (coord.), J ornadas sobre A rqueol ogía, H istoria y A rquitectura. Criterios de intervención en $A$ Patrimonio A rquitectónico, Logroño, pp. 11-34.

VALLE PÉREZ J. C., 1988, Análisis gliptográfico de la iglesia del monasterio de A rmenteira. Propuestas metodológicas, en A ctas de V Coloquio Internacional de G liptografía, Pontevedra, vol. 1, pp. 265-282.

Vela Cossío F., 1999, Intervenciones arqueológicas en proyectos de restauración de edificios históricos, en Tratado de rehabilitación, tomo 2: M etodol ogía de la restauración y de la rehabilitación, Madrid, pp. 211-217.

VIGIL-EsCALERA GUIRADO A., 2000, Cabañas de época visigoda: evidencias arqueológicas del sur de Madrid. Tipología, elementos de datación y discusión, A rchivo Español de A rqueología, LXX III. N úms. 181-182, pp. 223252.

W ESTMAN A., 2000, Upright stratigraphy: understanding and recording buildings in London, en Rosk Ams S. (eds.), Interpreting Stratigraphy. Site evaluation, recording procedures and stratigraphic analysis. Papers presented to the Interpreting Stratigraphy Conferences 1993-1997, British Archaeological Reports, International Series 910, 0xford, pp. 107-112.

W OOD J., 1994, B uilding A rchaeol ogy. A pplications in Practice, Oxford. 